\title{
High discrepancy in thrombotic events in non-small cell lung cancer patients with different genomic alterations
}

\author{
Yiwei Liu ${ }^{1 \#}$, Wanying Wang ${ }^{1 \#}$, Fengying Wu ${ }^{1}$, Guanghui Gao ${ }^{1}$, Jian $\mathrm{Xu}^{1}$, Xuefei $\mathrm{Li}^{2}$, Chao $\mathrm{Zhao}^{2}$, \\ Shuo Yang ${ }^{1}$, Shiqi Mao ${ }^{1}$, Yingying Pan ${ }^{1}$, Keyi Jia ${ }^{1}$, Chuchu Shao ${ }^{1}$, Bin Chen ${ }^{1}$, Shengxiang Ren ${ }^{1}$, Caicun Zhou ${ }^{1}$ \\ ${ }^{1}$ Department of Medical Oncology, Shanghai Pulmonary Hospital, School of Medicine, Tongji University, Shanghai, China; ${ }^{2}$ Department of Lung \\ Cancer and Immunology, Shanghai Pulmonary Hospital, School of Medicine, Tongji University, Shanghai, China \\ Contributions: (I) Conception and design: S Ren, Y Liu, W Wang; (II) Administrative support: S Ren, C Zhou; (III) Provision of study materials or \\ patients: B Chen, F Wu, G Gao, J Xu, X Li, C Zhou; (IV) Collection and assembly of data: Y Liu, W Wang, S Yang, S Mao, K Jia, Y Pan, C Shao; (V) \\ Data analysis and interpretation: Y Liu, W Wang, S Mao; (VI) Manuscript writing: All authors; (VII) Final approval of manuscript: All authors. \\ \#These authors contributed equally to this work. \\ Correspondence to: Prof Shengxiang Ren. Department of Medical Oncology, Shanghai Pulmonary Hospital \& Institute of Thoracic Cancer, School \\ of Medicine, Tongji University, No. 507, Zheng Min Road, Shanghai 200433, China. Email: harry_ren@126.com; Dr. Bin Chen. Department of \\ Medical Oncology, Shanghai Pulmonary Hospital \& Institute of Thoracic Cancer, School of Medicine, Tongji University, No. 507, Zheng Min \\ Road, Shanghai 200433, China. Email: binchen629@126.com.
}

Background: Acute complications, such as venous thromboembolism (VTE), are common in patients with advanced severe lung cancers. However, current VTE risk scores cannot adequately identify highrisk patients with non-small cell lung cancer (NSCLC). The study proposed to elucidated the incidence of thromboembolism (TE) in patients with different oncogenic aberrations and the impact of these aberrations on the efficacy of targeted therapy in patients with NSCLC.

Methods: A systemic review was conducted in Web of Science, PubMed, Embase and the Cochrane Library to evaluate the incidence of TE in different molecular subtypes of NSCLC. Data from patients diagnosed of advanced NSCLC who harboring anaplastic lymphoma kinase (ALK) or ROS proto-oncogene 1 receptor tyrosine kinase (ROS1) rearrangements since 2016 to 2019 were also retrospectively collected. A meta-analysis with random-effects model, sensitivity analysis and publication bias were performed. The principal summary measure was incidence of thrombotic events in NSCLC patients. And the efficacy of tyrosine kinase inhibitor (TKI) therapy was compared between the two subgroups.

Results: A total of 5,767 cases from 20 studies were included in the analysis of the incidence of thrombosis in patients with different oncogenic alterations. The pooled analysis showed a higher risk of thrombosis in ROS1fusion types (41\%, 95\% CI: 35-47\%) and ALK-fusion types (30\%, 95\% CI: 24-37\%) than in EGFR-mutation (12\%, 95\% CI: 8-17\%), KRAS-mutation (25\%, 95\% CI: 13-50\%), and wild-type (14\%, 95\% CI: 10-20\%) cases. A high prevalence of thrombosis (ALK: 24.4\%; ROS1: 32.6\%) was observed in the Shanghai Pulmonary Hospital (SPH) cohort of 224 patients with ALK or ROS1 fusion. Furthermore, patients with embolism had significantly shorter progression-free survival (PFS) after TKI therapy than those without embolism, both in the ALK+ cohort (5.6 vs. 12.9 months, $\mathrm{P}<0.0001$ ) and in the ROS1+ cohort (9.6 vs. 17.6 months, $\mathrm{P}=0.0481$ ).

Conclusions: NSCLC patients with ALK/ROS1 rearrangements are more likely to develop thrombosis than patients with other oncogenic alterations. Thrombosis may also be associated with an inferior response and PFS after TKI therapy.

Keywords: Non-small cell lung cancer (NSCLC); anaplastic lymphoma kinase (ALK); ROS1; thromboembolism (TE)

Submitted Sep 24, 2020. Accepted for publication Mar 23, 2021.

doi: $10.21037 /$ tlcr-20-1290

View this article at: http://dx.doi.org/10.21037/tlcr-20-1290 


\section{Introduction}

Acute complications, such as venous thromboembolism (VTE) and central airway obstruction, are common in advanced severe lung cancer and are associated with decreased performance status. VTE, which includes pulmonary embolism, deep-vein thrombosis, and migratory thrombophlebitis, is a common cancer-associated complication (1). The incidence of VTE reported in lung cancer varied in 5-14\% (2-4). Treatment choices for patients who experience embolism are limited, and survival is poor $(5-8)$.

The American Society of Clinical Oncology (ASCO)/ National Comprehensive Cancer Network (NCCN) guidelines routinely recommend assessment of VTE risk in patients with cancer $(9,10)$. However, many current clinical cancer-associated VTE risk assessment measures such as the Caprini score, the Khorana risk score (KRS), the CAMPASS-CAT score, and the Vienna Cancer and Thrombosis score (CATS) have low accuracy and poor consistency for identifying patients with NSCLC who are at high risk of VTE (11-13). Further, the reliability of these VTE risk assessment tools to distinguish patients who may potentially benefit from thromboprophylaxis is limited. Several randomized clinical trials $(14,15)$ and pooled analyses (16) have shown that a high percentage of cancer patients require anticoagulants, but this is associated with a 1.96-fold increase in the risk of bleeding. Therefore, an alternative VTE risk assessment tool is needed in clinical practice.

Carcinogenic driven mutations can stimulate complex cross signal transduction pathways, leading to uncontrolled growth, proliferation and survival of tumor cells. Due to the pathway of antagonizing different molecular targets is specific, treatment strategy based on different molecular profiling of tumor is precise and individualized (17). Recent studies have shown high discrepancy in the incidence of thrombotic events (TE) between NSCLC patients with different genomic alterations (18-24). Two studies showed the incidence of TE in NSCLC patients who harbored epidermal growth factor receptor (EGFR) mutations to be $9.0-24.8 \%$ $(23,24)$, whereas the incidence of TE in NSCLC patients with Kirsten rat sarcoma viral oncogene homolog (KRAS) mutation ranged from $21.6-54.8 \%(18,24)$. In contrast to EGFR/KRAS-mutated NSCLC, several studies have reported a higher incidence of TE in anaplastic lymphoma kinase (ALK) or ROS proto-oncogene 1 receptor tyrosine kinase (ROS1) rearrangements-positive patients (19-22,25).
Therefore, a pooled analysis is urgently needed to determine the incidence of VTE in high-risk populations on the basis of the molecular features of NSCLC, which in turn will allow a more personalized approach for patients with NSCLC, rather than the broad screening currently used.

In view of the negative impact of VTE formation on mortality, poor prognosis has also been observed in cancer patients receiving chemotherapy $(5,26)$. Recent observational studies have reported conflicting results of the impact of VTE on overall survival. Two studies $(20,27)$ found that VTE was associated with a higher risk of death, with an almost two-fold increase in risk for ALK/ROS1 $1^{+}$patients who had experienced thromboembolic events. However, another recent study found no evidence of an inferior overall survival rate (19), partly accounted by the insufficient duration of follow-up and limited sample size. Alexander et al. (28) demonstrated a numerical lower ORR to TKI treatment in ROS1+ NSCLC cases from multicenter in Australia. Due to its small size, there was a need to explore the efficacy of TKI in such oncogene-driven NSCLC patients. It is noteworthy that there is a lack of data on whether the formation of thrombosis impacts the clinical response to tyrosine kinase inhibitor (TKI) therapy. Therefore, we enrolled an ALK/ ROS1-positive cohort from Shanghai Pulmonary Hospital (SPH) in order to explore the response to TKI therapy in ALK/ROS1 NSCLC patients with TE.

We carried out a meta-analysis of 5,767 cases from 20 studies to comprehensively investigate the risk of thrombosis in NSCLC subgroups with different oncogenomic alterations and to provide guidance for early thromboprophylaxis. Aiming to compare the clinical outcome to TKI treatment in patients with thrombosis, we validated the results of our meta-analysis in a cohort of 224 patients with ALK/ ROS1 fusion from SPH. We present the following article in accordance with the PRISMA reporting checklist (available at http://dx.doi.org/10.21037/tlcr-20-1290).

\section{Methods}

\section{Search strategy and selection criteria}

We conducted a systemic review in following databases: Web of Science, PubMed, Embase and the Cochrane Library, in order to find studies on the prevalence of thrombotic events in oncogene-driven NSCLC. Studies published up to June 2020 were considered, and there were no language restrictions. Major conference proceedings including the European Society for Medical Oncology, 
the American Society of Clinical Oncology, and the World Conference of Lung Cancer were also reviewed based on abstracts and presentations up to June 2020 . Keywords for the literature search included: (Thrombosis OR Thrombophlebitis OR Embolism OR Thromboses OR Thromboembolism) AND (NSCLC OR Carcinoma, NonSmall-Cell Lung OR Carcinoma, Non Small Cell Lung OR Carcinomas, Non-Small-Cell Lung OR Lung Carcinoma, NonSmall-Cell OR Lung Carcinomas, Non-Small-Cell OR NonSmall-Cell Lung Carcinomas OR Nonsmall Cell Lung Cancer OR Non-Small-Cell Lung Carcinoma OR Non Small Cell Lung Carcinoma OR Carcinoma, Non-Small Cell Lung OR NonSmall Cell Lung Cancer). We carried out the analysis in compliance with the PRISMA 2020 Statement (29).

The studies which met following criteria were enrolled in the analysis: (I) studies which assessed the prevalence of thrombotic events in NSCLC; (II) available genomic alteration information; (III) sufficient subgroup analysis according to clinicopathologic features. Reviews, case reports, irrelevant studies, and incomplete studies were excluded, as were studies for which gene alteration information was unavailable. If data were replicated in several studies, then the most recent and complete reports were included. Two different reviewers (Y.L. and W.W.) independently assessed the articles in the reference list based on title/abstracts and full text of potentially enrolled articles in accordance with above criteria. If there was a difference in opinion, an agreement was reached through discussion with a third reviewer (S.M.).

\section{Data extraction and methodological quality assessment}

Two authors (Y.L. and W.W.) independently excerpted the following data from the enrolled studies: name of first author, trial name, publication year, study population, number of patients enrolled, incidence of thrombosis, and prevalence of TE grouped according to genomic characteristics. Disagreements were resolved through discussion with a third reviewer (S.M.).

As previously reported (30), an Agency for Healthcare Research and Quality (AHRQ) 11-item checklist was used to evaluate the methodological quality of the studies included. In specific, the scored ' 0 ' indicated ' $\mathrm{NO}$ ' or 'UNCLEAR'; and score ' 1 ' indicated 'YES'. The quality was defined as follows: $8-11=$ high; $4-7=$ moderate; $0-3=$ low. The quality evaluation was independently conducted by two evaluators (Y.L. and W.W.), and if there was a difference of opinion, an agreement was reached based on the opinion of a third evaluator (S.M.).

\section{Patient enrollment}

Patients diagnosed with ALK or ROS1-fusion NSCLC at the Shanghai Pulmonary Hospital (SPH), Tongji University, were enrolled in this study between January 1, 2016 and December 31, 2019. Patient clinicopathological characteristics including demographic characteristics, pathological features, and molecular diagnosis, as well as the tumor, node, and metastasis (TNM) stage (version 8) were collected. We obtained the written informed consent from all participants. This study was approved by the Ethics Committee of the Shanghai Pulmonary Hospital, Tongji University School of Medicine. All procedures performed in this study involving human participants were in accordance with the Declaration of Helsinki (as revised in 2013).

\section{Definition of venous thrombotic events}

All VTEs, including those that were symptomatic or incidentally discovered on imaging, were reviewed and recorded. All VTEs were confirmed on the basis of ultrasound, computed tomography (CT), or magnetic resonance imaging (MRI). In the analyses, VTE including pulmonary embolus (PE) and deep venous thrombosis (DVT) were comprehensive analyzed regardless of clinical symptoms (symptomatic versus incidental) or location, because recent study no prognostic difference or risk of recurrence between cancer patients with distal and proximal DVT, or between those with subsegmental PE and segmental PE.

\section{Driver mutation analysis}

As described in our previous studies (31-33), the mutation status of the patients was analyzed using the amplification refractory mutation system (Amoy Diagnostics Co Ltd., Xiamen, China).

\section{Statistical analysis}

For the meta-analysis, statistical heterogeneity was assessed using $\chi^{2}$ test, and heterogeneity across trials was assessed using the $\mathrm{I}^{2}$ statistic. Low-level heterogeneity was defined as $\mathrm{P}>0.1$ of the $\chi^{2}$ test and $\mathrm{I}^{2}<25 \%$. Fixed-effect models were used in pooled analysis if there was no statistically significant heterogeneity; otherwise, a random-effects model was used. A sensitivity analysis was conducted through exclusion 


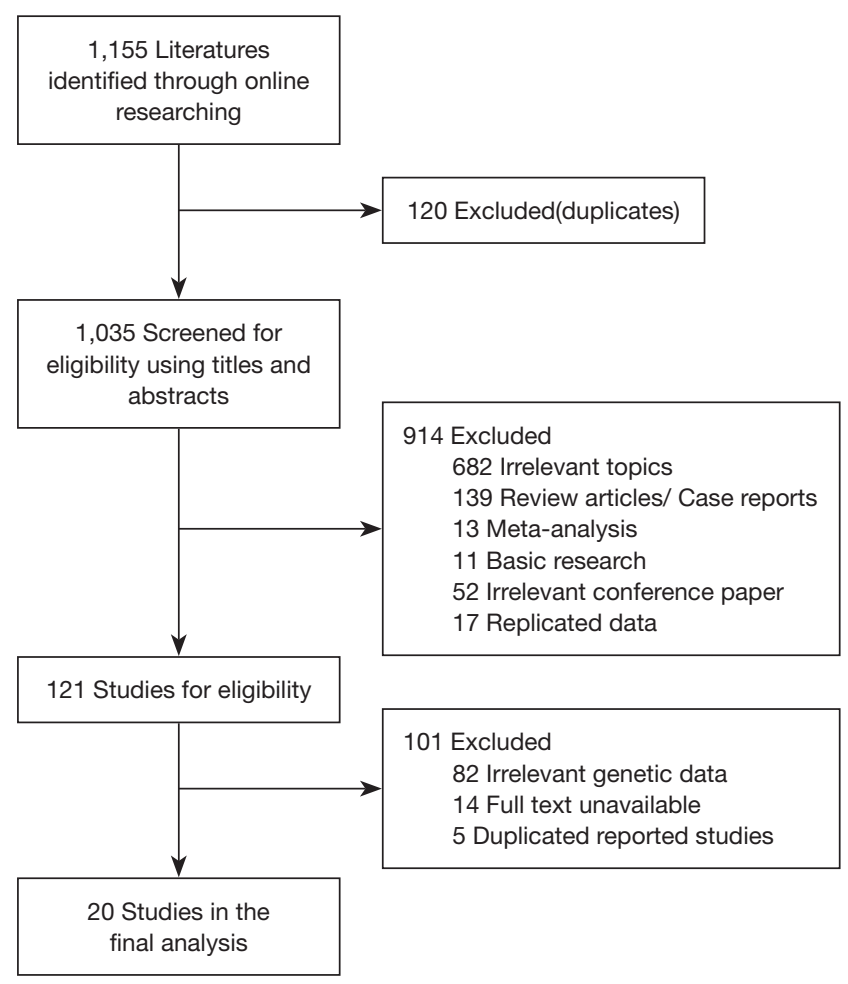

Figure 1 Flowchart of the identification of eligible studies for the meta-analysis.

of each single enrolled study to determine the stability of the overall results. A possible publication bias was evaluated. The principal summary measure was incidence of thrombotic events in NSCLC patients. Statistical analysis and plots were performed using $\mathrm{R}$ software, version 4.0.1 (https://www.r-project.org/). The Begg's, Egger's tests and Peter's tests were used to assess publication bias. A P value $<0.10$ indicated significant asymmetry and publication bias.

Statistical analyses of the SPH patient cohort were performed in SPSS statistics software (version 21.0, IBM Corp, Armonk, NY, USA, RRID:SCR_019096). Differences in clinical characteristics between the TE and non-TE groups were analyzed using Student's $t$ test, the $\chi^{2}$ test, or Fisher's exact test. Survival was analyzed through the log-rank test in GraphPad Prism (Version 6, RRID:SCR_002798). Statistical significance was defined as $\mathrm{P}<0.05$. All tests were two-tailed.

\section{Results}

\section{Characteristics of the included studies}

The flowchart in Figure 1 shows the selection process for the included studies. A total of 1,155 publications were initially reviewed, and 20 studies were enrolled in the metaanalysis after qualification (18-25,27,28,34-43). Through preliminary screening, 121 studies met many but not all inclusion criteria. However, they were finally excluded for the reason that 82 studies lacked complete genetic data, 14 studies were unavailable for full text and 5 studies were duplicated reported. In total, 5,767 cases from 20 studies that explored the incidence of thrombosis in different oncogenedriven lung cancers were analyzed. The main characteristics of the included studies are presented in Table 1. All the included studies were published between 2014 and 2020 . Four studies were conducted in Asia, five in North America, two in Australia, six in Europe, one study covered multiple countries, and for two studies, the country of publication was unknown. The methodological quality assessment based on AHRQ score were listed in Table S1.

Exclusion of each enrolled studies did not significantly affect the pooled result of incidence of TE in lung cancer patients (Figure S1A). Publication bias was assessed through the Begg's funnel plot and the Egger's or Peter's test (Figure S1B), and was only detected for the incidence of TE in the ROS1 fusion subgroup $(\mathrm{P}<0.05$ assessed by Egger's test). After advance analysis, the Begg's funnel plot was symmetric, which indicated no evidence of publication bias (Figure S1C).

\section{Prevalence of thrombotic events and their association with mutation status}

Overall, 5,767 NSCLC patients who underwent driver gene test from 20 studies were included in this metaanalysis. The incidence of thrombosis in these studies ranged from $3.9-47.6 \%$, reflecting the high heterogeneity of TE incidence across the studies. In this meta-analysis, the overall incidence of TE was $23 \%$ (95\% CI: 17-28\%) and significant heterogeneity was evident $\left(\mathrm{I}^{2}=96 \%, \mathrm{P}<0.01\right.$, Figure 2). Therefore, subgroup meta-analysis was stratified by mutation status.

As shown in Figure 3, mutation status partly accounted for the heterogeneity. The pooled results for most subgroups were markedly changed when stratified by the specific mutation status. The pooled prevalence of TE was significantly higher in patients with an ALK/ ROS1 fusion. For the ROS1-fusion NSCLC group, the pooled incidence of thrombosis was $41 \%$ (95\% CI: $35-47 \%)$ and low heterogeneity was found $\left(\mathrm{I}^{2}=3 \%\right.$, $\mathrm{P}=0.38$, Figure $3 B$ ). Similarly, in the ALK-fusion NSCLC 
Table 1 Characteristics of studies enrolled in the meta-analysis

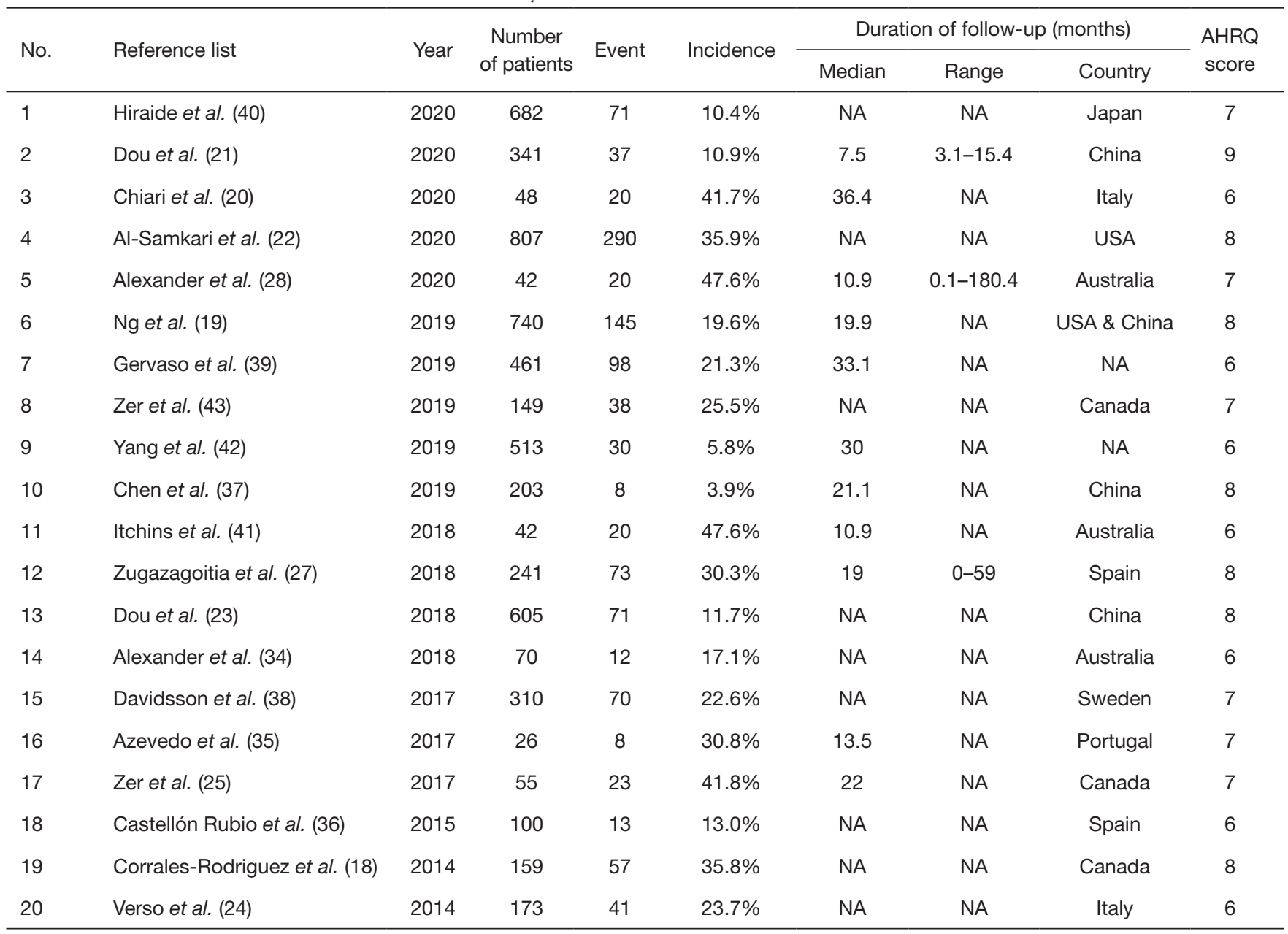

group, the overall incidence of TE was 30\% (95\% CI: $24-37 \%)$, with moderate heterogeneity $\left(\mathrm{I}^{2}=78 \%, \mathrm{P}<0.01\right.$, Figure $3 A$ ). Among patients with EGFR mutations, the overall incidence was $12 \%$ (95\% CI: $8-17 \%$ ), with high heterogeneity. For the KRAS-mutant subsets, the pooled prevalence was $25 \%$ (95\% CI: $13-50 \%)$, with high heterogeneity. In the non-EGFR/ALK/ROS1 population, the prevalence of thrombosis was $14 \%$ (95\% CI: 10-20\%), and high heterogeneity was found.

\section{Clinical characteristics and incidence of thrombosis in the SPH cohort}

Based on the findings that $\mathrm{ALK} / \mathrm{ROS} 1^{+}$patients were in higher risk of TE formation, which was observed in metaanalysis, we validated the findings of our meta-analysis in a cohort of 224 patients with ALK or ROS1 fusion from SPH, and compared the efficacy of TKI therapy between VTE and non-VTE ALK/ROS1-fusion NSCLC patients. The flowchart of the patient selection process is presented in Figure 4. Table 2 summarizes the patients' clinical characteristics and previous anti-tumor treatments. Representative images of ultrasound and computed tomography were provided for the diagnosis of VTE (Figure S2). The SPH cohort included 135 patients with ALK fusion and 89 with ROS1 fusion. Of the total 224 patients, $62(27.7 \%)$ developed thrombosis. The incidence of VTE was $24.4 \%$ (33 of 135 ) and $32.6 \%$ (29 of 89) for ALKfusion and ROS1-fusion NSCLC patients, respectively. This analysis included 175 patients who received TKI therapy. Of these patients, 49 (28.0\%) experienced VTE formation during the time they were receiving TKI treatment. In particular, the incidence of VTE was $21.6 \%$ and $44.0 \%$ for patients who received TKI therapy in the ALK and ROS1- 


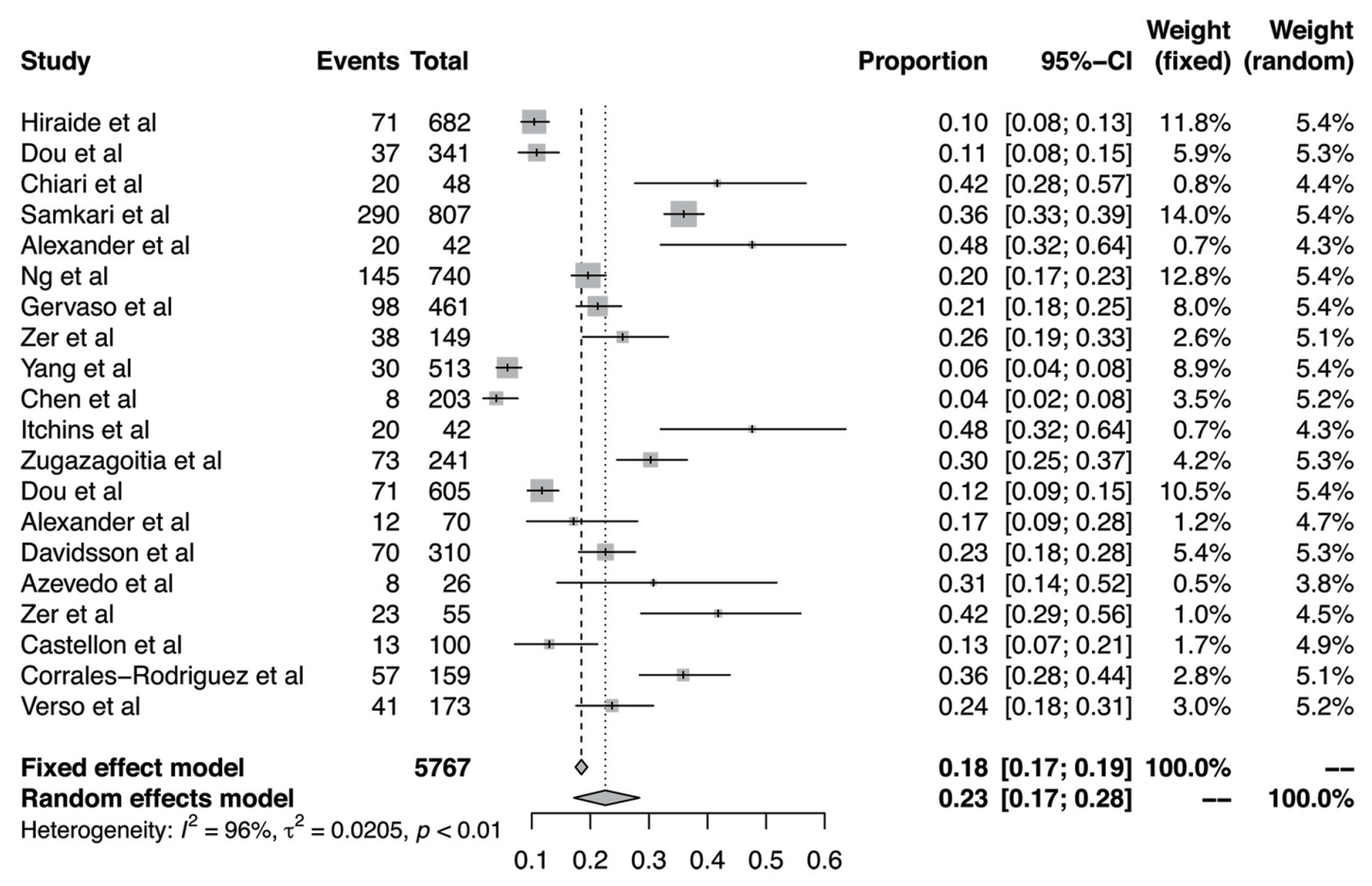

Figure 2 Meta-analysis (forest plot) of the prevalence of thrombotic events in lung cancer patients.

fusion NSCLC groups, respectively. The majority of the included patients were non-smokers and had stage IV adenocarcinoma. Most patients had received TKI therapy as a first-line treatment. There was no significant difference in the clinical characteristics between the TE and non-TE groups.

\section{The impact of thrombosis on the efficacy of ALK/ROS1-TKIS}

In the ALK-positive cohort, 125 patients received TKI therapy including 27 patients with TE and 98 without TE. Overall, patients with TE showed a numerically lower objective response rate (ORR) compared to patients without TE $(66.7 \%$ vs. $77.6 \%, \mathrm{P}=0.246)$ (Figure $5 A)$. TE patients also showed significantly shorter progression-free survival (PFS) compared to patients without TE (5.6 vs. 12.9 months, $\mathrm{P}<0.0001$; HR: 3.320, 95\% CI: $1.889-5.833$ ) (Figure 5B). Among the ALK fusion subgroup, 95 (76.0\%) patients received crizotinib and 30 (24.0\%) received alectinib treatment. Among the patients treated with crizotinib, TE was associated with a numerically lower ORR (65.2\% vs. $75.0 \%, \mathrm{P}=0.360$ ) (Figure $\mathrm{S} 3 \mathrm{~A}$ ) and a significantly shorter PFS (HR: 4.960; 95\% CI: 2.627-9.365; $\mathrm{P}<0.0001$ ) (Figure S3B).
Among the patients who received alectinib, a numerically lower ORR (75.0\% vs. 84.6\%, $\mathrm{P}=0.538$ ) (Figure S3C) and shorter PFS were also observed (12.17 vs. NR, HR: 1.238, 95\% CI: 0.270-5.670; $\mathrm{P}=0.78$ ) (Figure S3D).

In the ROS1 fusion subgroup, 50 patients received crizotinib including 22 patients with TE and 28 patients without TE. Patients with TE showed a numerically lower ORR compared to those without TE (64.3\% vs. $31.8 \%$, $\mathrm{P}=0.082$ ) (Figure $5 C$ ). A significantly shorter PFS was also observed in patients with TE (9.6 vs. 17.6 months; HR: 2.270, 95\% CI: 0.998-5.215; $\mathrm{P}=0.0481$ ) (Figure 5D).

\section{Discussion}

In this study, a high discrepancy was found in the incidence of VTE in patients with NSCLC with different genomic alterations. The pooled prevalence of TE occurrence was $41 \%$ and $30 \%$ for ROS1 or ALK-positive NSCLC patients, respectively. However, the incidence of TE was lower in EGFR/KRAS-mutant or wild-type NSCLC patients (incidence: $12 \%, 25 \%, 14 \%$, respectively). The high incidence of TE was further validated in our SPH cohort. Moreover, in the SPH cohort, ROS1/ALK-fusion NSCLC patients who experienced TE showed a two-fold increase in 
A

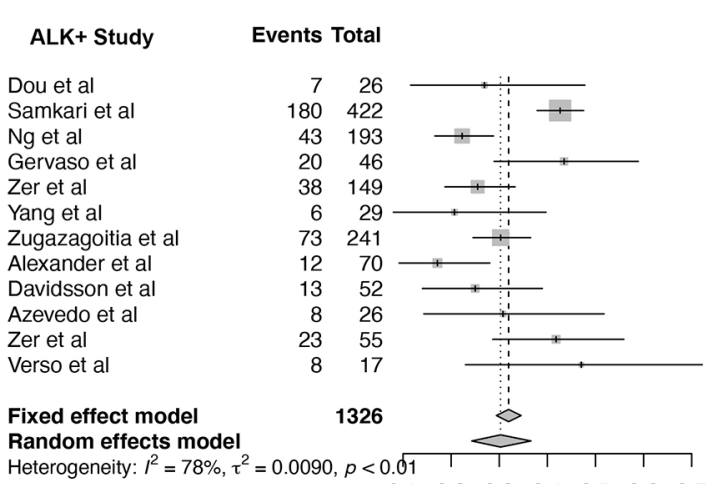

Weight Weight

Proportion 95\%-Cl (fixed) (random)

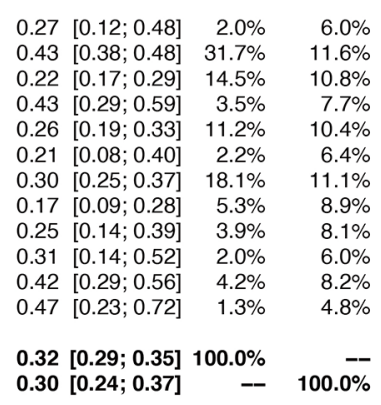

$0.30[0.24 ; 0.37] \quad-\quad 100.0 \%$

$\begin{array}{lllllll}0.1 & 0.2 & 0.3 & 0.4 & 0.5 & 0.6 & 0.7\end{array}$

B

ROS1+ Study

Events Total

Chiari et al

Alexander et al

$\mathrm{Ng}$ et al

Itchins et al

Fixed effect model

Random effects model

Heterogeneity: $I^{2}=3 \%, \tau^{2}=0.0001, p=0.38$

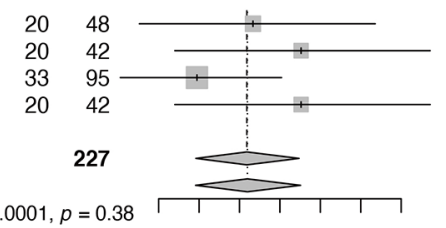

C KRAS+ Study

0.30 .350 .40 .450 .50 .550 .6

Events Total

$\mathrm{Ng}$ et al

Dou et al

Corrales-Rodriguez et al

Verso et al

Fixed effect model

Random effects mode

Heterogeneity: $l^{2}=89 \%, \tau^{2}$

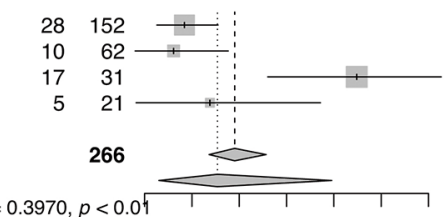

D EGFR+ Study

Events Total

$\begin{array}{lllllll}0.1 & 0.2 & 0.3 & 0.4 & 0.5 & 0.6 & 0.7\end{array}$

Hiraide et al
$\mathrm{Ng}$ et al
Gervaso et al
Yang et al
Chen et al
Dou et al
Davidsson et al
Corrales-Rodriguez et al
Verso et al

Verso et al

Fixed effect model

Random effects model Heterogeneity: $I^{2}=85 \%, \tau^{2}=0.2817, p<0.01$

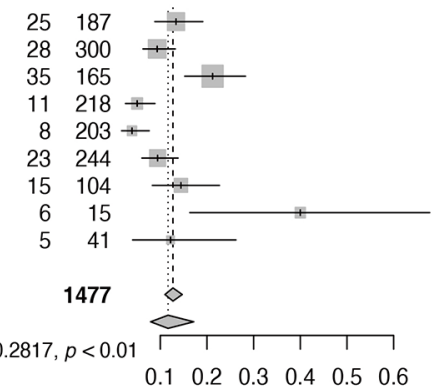

E WT Study

Events Total

$\begin{array}{llllll}0.1 & 0.2 & 0.3 & 0.4 & 0.5 & 0.6\end{array}$

Hiraide et al

Dou et al

Samkari et al

Gervaso et al

Yang et al

Dou et al

Davidsson et al

Castellon et al

Corrales-Rodriguez et al

Fixed effect model

Random effects mode

Random effects model

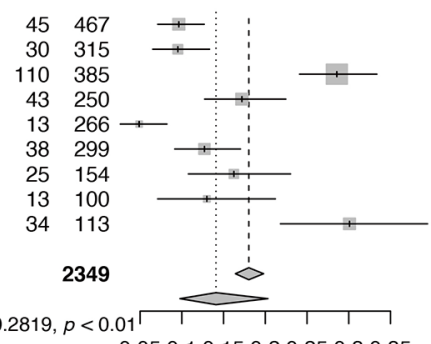

$0.42[0.28 ; 0.57] 21.1 \% \quad 21.3 \%$ $0.48[0.32 ; 0.64] 18.5 \% \quad 18.7 \%$ $0.35[0.25 ; 0.45] \quad 41.9 \% \quad 41.2 \%$ $0.48[0.32 ; 0.64] \quad 18.5 \% \quad 18.7 \%$

$0.41[0.35 ; 0.47] 100.0 \%$

Weight Weight

Proportion 95\%-Cl (fixed) (random)

$0.18[0.13 ; 0.26] \quad 37.9 \% \quad 27.3 \%$ $0.16[0.08 ; 0.28] \quad 13.2 \% \quad 24.2 \%$ $0.55[0.36 ; 0.73] \quad 41.6 \% \quad 27.4 \%$ $0.24[0.08 ; 0.47] \quad 7.3 \% \quad 21.1 \%$

$\begin{array}{llr}0.29[0.24 ; 0.36] & 100.0 \% & -- \\ 0.25[0.13 ; 0.50] & - & 100.0 \%\end{array}$

$0.25[0.13 ; 0.50] \quad-100.0 \%$

Proportion $\quad 95 \%-\mathrm{Cl}$ (fixed) (random)

$0.13[0.09 ; 0.19] \quad 15.8 \% \quad 12.3 \%$ $0.09[0.06 ; 0.13] \quad 16.9 \% \quad 12.4 \%$ $0.21[0.15 ; 0.28] \quad 24.3 \% \quad 12.8 \%$ $0.05[0.03 ; 0.09] \quad 6.3 \% \quad 10.6 \%$ $0.04[0.02 ; 0.08] \quad 4.6 \% \quad 9.7 \%$ $0.09[0.06 ; 0.14] \quad 13.9 \% \quad 12.1 \%$ $0.14[0.08 ; 0.23] \quad 9.6 \% \quad 11.5 \%$ $0.40[0.16 ; 0.68] \quad 5.5 \% \quad 10.2 \%$ $0.12[0.04 ; 0.26] \quad 3.1 \% \quad 8.5 \%$

$0.13[0.11 ; 0.15] 100.0 \%$ $0.12[0.08 ; 0.17] \quad-\quad 100.0 \%$ Proportion $\quad 95 \%-\mathrm{Cl} \begin{gathered}\text { Weight } \\ \text { (fixed) }\end{gathered} \begin{array}{r}\text { Weight } \\ \text { (random) }\end{array}$

$0.10[0.07 ; 0.13] \quad 11.3 \% \quad 11.5 \%$ $0.10[0.07 ; 0.13] \quad 7.5 \% \quad 11.1 \%$ $0.29[0.24 ; 0.33] \quad 35.0 \% \quad 12.1 \%$ $0.17[0.13 ; 0.22] \quad 11.8 \% \quad 11.6 \%$ $0.05[0.03 ; 0.08] \quad 3.1 \% \quad 9.8 \%$ $0.13[0.09 ; 0.17] \quad 9.9 \% \quad 11.4 \%$ $0.16[0.11 ; 0.23] \quad 6.8 \% \quad 11.0 \%$ $0.13[0.07 ; 0.21] \quad 3.4 \% \quad 10.0 \%$ $0.30[0.22 ; 0.39] \quad 11.1 \% \quad 11.5 \%$

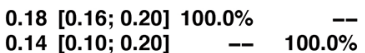

Proportion $\quad 95 \%-\mathrm{Cl}$ (fixed) (random)

0.050 .10 .150 .20 .250 .30 .35

Figure 3 Subgroup analyses of the prevalence of thrombosis based on patient's mutation status. (A) ALK-fusion; (B) ROS1-fusion; (C) KRAS-mutant; (D) EGFR-mutant; (E) wild-type. ALK, anaplastic lymphoma kinase; EGFR, epidermal growth factor receptor; KRAS, Kirsten rat sarcoma viral oncogene homolog; ROS1, ROS pro-oncogene 1 receptor tyrosine kinase. 


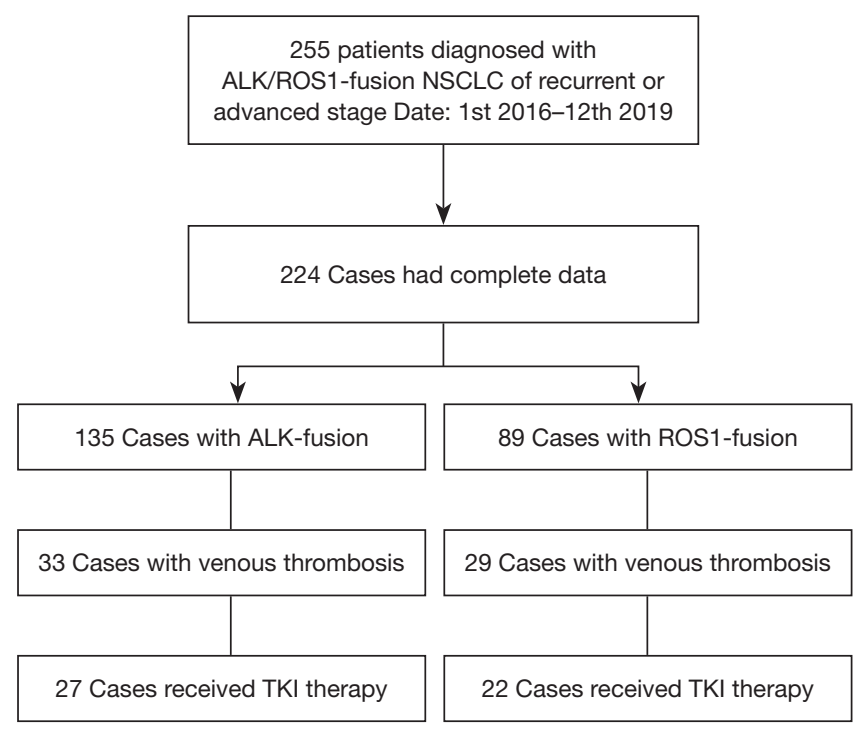

Figure 4 Flowchart showing the selection of eligible patients.

the risk of tumor progression in response to TKIs compared to those without TE. Taken together, results of the present study suggest that the molecular profiles of NSCLC should be incorporated into a VTE risk score and decision-making algorithm for VTE diagnosis and prophylaxis.

Patients with lung cancer have a high risk of VTE(44), and TE is a major cause of morbidity and mortality (45-47). Therefore, many clinical cancer-associated VTE risk assessment measures have been devised to accurately identify patients at risk; these tools include the Caprini score, the Khorana risk score (KRS), the CAMPASS-CAT score, and the Vienna Cancer and Thrombosis score (CATS). Based on current studies, above score systems showed limited specificity or accuracy or calibration in identify cases of VTE risk. In previous studies, Caprini VTE risk model was able to predict the occurrence of VTE in $10.3 \%$ of postoperative lung cancer patients, but with an accuracy of only $61.6 \%$ (13). Therefore, there may be some evaluation bias if Caprini VTE risk model was used in advanced lung cancer patients. Low accuracy and evaluation bias limited the application of the model in patients with advanced lung cancer. Secondly, as for KRS score, the retrospective study which enrolled lung cancer demonstrated sensitivity as $10 \%$ and specificity as $100 \%$ for patients with high KRS score (48). A meta-analysis confirmed a higher VTE rate of $11 \%$ in patients with high-risk KRS than a low-/ intermediate-risk or intermediate-risk score (low-risk: 5.0\%; intermediate-risk: 6.6\%) (12). Therefore, low-moderate KRS group still have substantial residual VTE risk. KRS's
Table 2 Baseline characteristics of patients enrolled in the retrospective cohort

\begin{tabular}{|c|c|c|c|}
\hline Characteristic & VTE $(n=49)$ & Non-VTE $(n=126)$ & $P$ value \\
\hline Age & & & 0.182 \\
\hline Year, mean \pm SD & $54 \pm 12$ & $52 \pm 12$ & \\
\hline Sex & & & 0.892 \\
\hline Male & $22(44.9 \%)$ & $58(46.0 \%)$ & \\
\hline Female & 27 (55.1\%) & $68(54.0 \%)$ & \\
\hline Smoking & & & 0.475 \\
\hline Smoker & $4(8.2 \%)$ & $15(11.9 \%)$ & \\
\hline Non-smoker & 45 (91.8\%) & $111(88.1 \%)$ & \\
\hline Pathology & & & 0.789 \\
\hline ADC & $40(81.6 \%)$ & $105(83.3 \%)$ & \\
\hline NSCLC & $9(18.4 \%)$ & $21(16.7 \%)$ & \\
\hline TNM stage & & & 0.459 \\
\hline IIIB-C & $7(14.0 \%)$ & $13(10.3 \%)$ & \\
\hline IV & $42(86.0 \%)$ & $113(89.7 \%)$ & \\
\hline ECOG PS & & & 0.187 \\
\hline 0 & $0(0.0 \%)$ & $6(4.8 \%)$ & \\
\hline 1 & 49 (100.0\%) & $120(95.2 \%)$ & \\
\hline Line of TKI & & & 0.072 \\
\hline 1 & $40(81.6 \%)$ & $115(91.3 \%)$ & \\
\hline$\geq 2$ & $9(18.4 \%)$ & $11(8.7 \%)$ & \\
\hline Gene alteration & & & 0.003 \\
\hline ALK & 27 (55.1\%) & $98(77.8 \%)$ & \\
\hline ROS1 & 22 (44.9\%) & $28(22.2 \%)$ & \\
\hline TKI & & & 0.049 \\
\hline Crizotinib & $45(91.8 \%)$ & $100(79.4 \%)$ & \\
\hline Alectinib & $4(8.2 \%)$ & $26(20.6 \%)$ & \\
\hline
\end{tabular}

VTE, venous thromboembolism; NSCLC, non-small cell lung cancer; TKI, tyrosine kinase inhibitor.

ability to consistently discriminate high versus low VTE risk in lung cancer was suboptimal, and even within the highrisk group, when assessed by KRS score, the risk of VTE in lung cancer was underestimated compared to that in other cancers (12). Thirdly, for COMPASS-CAT scores, the large retrospective study demonstrated a sensitivity of $95 \%$ and a specificity of $12 \%$, but the calibration was poor according to the Hosmer-Lemeshow test (11). The consistency 

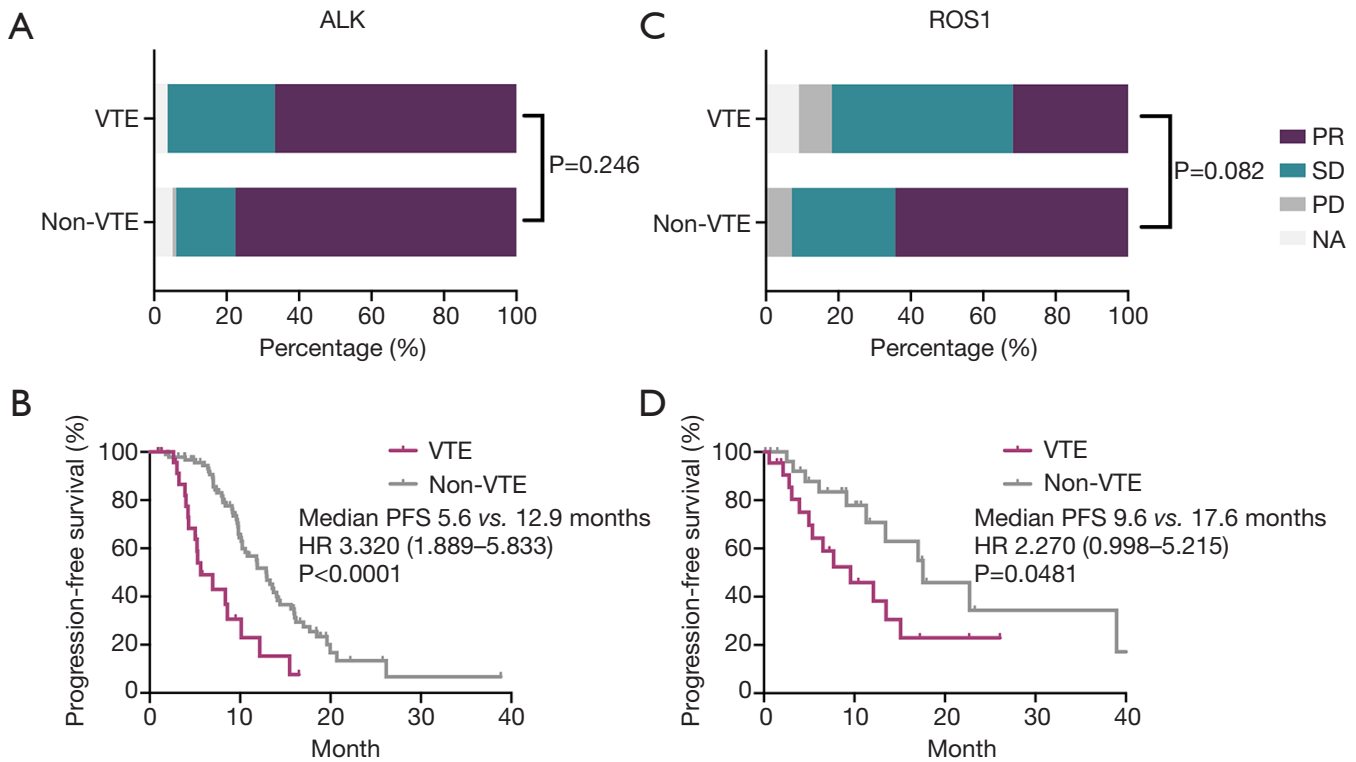

Figure 5 Comparison of TKI response and PFS between the VTE group and non-VTE group in the ALK-fusion and ROS1-fusion cohort, respectively. (A) Response to TKI therapy among ALK ${ }^{+}$patients; (B) PFS of ALK ${ }^{+}$patients treated with TKI therapy; (C) response to TKI therapy among $\mathrm{ROS}^{+}$patients; (D) PFS of $\mathrm{ROS}^{+}$patients treated with TKI therapy. ALK, anaplastic lymphoma kinase; PFS, progressionfree survival; ROS1, ROS pro-oncogene 1 receptor tyrosine kinase; TKI, tyrosine kinase inhibitor; VTE, venous thromboembolis.

between the predicted risk by COMPASS-CAT model and the actual risk of VTE was poor. Fourthly, as for CATS, the prospective observational cohort study demonstrated a sensitivity of $19.1 \%$ at the cutoff point for highest risk (score $\geq 5$ ) and a specificity of $98.2 \%$ (49). In total, current VTE risk scoring model was limited in identifying lung cancer patients of high risk.

As far as we know, the present study is the first to use pooled analyses to examine the prevalence of VTE based on molecular status. We found a high risk of VTE in ALK/ ROS1-positve NSCLC patients compared with EGFR/ KRAS-mutant or wild-type NSCLC patients. In particular, in several recent retrospective studies, the prevalence of VTE was found to range from $28-42 \%$ in lung cancer patients with ALK rearrangements, which is 3 - to 5-fold higher than the prevalence of TE previously reported in advanced NSCLC patients (22,25). Additionally, Al-Samkari et al. (22) also found a significantly higher recurrence rate for VTE in ALK-positive patients (ALK $v$ s. $\mathrm{ALK}^{-}: 13.5 \%$ vs. $3.1 \%$ ), which increased the risk of mortality by 4.85 -fold. A high incidence of VTE in ALK-NSCLC patients was also demonstrated in a prospective cohort (21). For ROS1-fusion NSCLC patients, the incidence of VTE was previously reported as $34.7-48 \%$. A prospective trial also supported the high prevalence of VTE occurrence. VTE can occur in the peri-diagnostic period (19), in the tumor progression period, or during treatment, with an incidence of $32.1 \%$, $35.7 \%$, and $32.0 \%$, respectively (20). The prevalence of VTE was compared between $\mathrm{ALK}^{+}$and $\mathrm{ROS}^{+}$patients, and the latter group was found to be significantly more likely to develop VTE. Taken together, the incidence of TE was highly discrepant in patients with NSCLC who had different genomic alterations, and molecular profiles need to be incorporated into a VTE risk assessment tool in the future.

The possible underlying mechanism for the development of TE in ALK/ROS1-fusion lung cancer patients might be as follows. Firstly, it is possible that mucin abundance in these subsets of tumors generates various signals, which eventually results in the activation and aggregation of platelets, and subsequent embolization. Secondly, cooccurrence of prothrombotic mutations could be the underlying mechanism of VTE risk and the reason for inferior response to TKI treatment. Lee et al. (50) reported on two patients with co-mutated Factor V Leiden (FVL) who developed recurrent VTE. One of these cases was found to have an antithrombin III (ATIII) deficiency and a positive lupus anticoagulant test. Beside FVL mutation, the variant in F5 was reported to increase the risk of VTE by over 10 -fold (51). Thirdly, downstream signaling of 
the ALK/ROS1 alteration might create cross-talk with procoagulant factors. Among these factors, tissue factor (TF) could interact with the coagulation factor FVII, triggering subsequent thrombin formation. ALK/ROS1 downstream $\mathrm{NF}-\kappa \mathrm{B}$ signaling might be involved in the expression of TF (52). Sen et al. (53) found upregulation of TF in ALKfusion NSCLC patients.

The development of thrombosis is associated with significantly inferior prognosis. We further explored the clinical response to TKI therapy in patients with ALK/ ROS1-fusion. Significantly shorter PFS and a numerically lower ORR after TKI therapy were observed in the VTE group. In line with this finding, Lee et al. (50) reported one ROS1-positive case who rapidly developed VTE and progressed to TKI monotherapy. As for the impact on long-term survival, the occurrence of VTE was associated with a poor prognosis in ALK-NSCLC patients (HR for $\mathrm{OS}=2.88, \mathrm{P}=0.059$ ) (25), which suggests a potential role for thromboprophylaxis in ALK/ROS1NSCLC. One example that supports this is the case of a patient who received combined anticoagulant medication with crizotinib, resulting in VTE control and a durable remission of tumor (50).

This study has several limitations. Firstly, the majority of the included studies were retrospective with small sample sizes. Thus, our conclusions should be interpreted with caution. Secondly, publication bias might exist in this area of study. Thirdly, our SPH cohort was a retrospective study enrolled one clinical center, with only a small sample size of ALK/ROS1 $1^{+}$patients. Larger prospective trials will be needed for further validation.

\section{Conclusions}

A high level of discrepancy was found in the incidence of TE among patients with NSCLC with different genomic alterations, and an ALK/ROS1 rearrangement was associated with a higher incidence of TE. Additionally, patients who developed VTE showed an inferior response to TKI therapy, which suggests that molecular profiles need to be incorporated into future VTE risk assessment models.

\section{Acknowledgments}

Funding: This work was sponsored by grants from the National Natural Science Foundation of China (No. 81772467 to SR, No. 81972167 to SR), and the Shanghai
Pujiang Program (No. 2019PJD048 to SR).

\section{Footnote}

Reporting Checklist: The authors have completed the PRISMA reporting checklist. Available at http://dx.doi. org/10.21037/tlcr-20-1290

Data Sharing Statement: Available at http://dx.doi. org/10.21037/tlcr-20-1290

Conflicts of Interest: All authors have completed the ICMJE uniform disclosure form (available at http://dx.doi. org/10.21037/tlcr-20-1290). CZ has served as an unpaid editorial board member of Translational Lung Cancer Research from Aug 2020 to Jul 2022. The other authors have no conflicts of interest to declare.

Ethical Statement: The authors are accountable for all aspects of the work in ensuring that questions related to the accuracy or integrity of any part of the work are appropriately investigated and resolved. This study was approved by the Ethics Committee of the Shanghai Pulmonary Hospital, Tongji University School of Medicine. All procedures performed in this study involving human participants were in accordance with the Declaration of Helsinki (as revised in 2013). Written informed consent was obtained from all participants.

Open Access Statement: This is an Open Access article distributed in accordance with the Creative Commons Attribution-NonCommercial-NoDerivs 4.0 International License (CC BY-NC-ND 4.0), which permits the noncommercial replication and distribution of the article with the strict proviso that no changes or edits are made and the original work is properly cited (including links to both the formal publication through the relevant DOI and the license). See: https://creativecommons.org/licenses/by-nc-nd/4.0/.

\section{References}

1. Kuperman A, López-Reyes R, Bosco LJ, et al. Anemia and bleeding in patients receiving anticoagulant therapy for venous thromboembolism. J Thromb Thrombolysis 2018;45:360-8.

2. Tagalakis V, Levi D, Agulnik JS, et al. High risk of deep vein thrombosis in patients with non-small cell lung cancer: A cohort study of 493 patients. J Thorac Oncol 
2007;2:729-34.

3. Kadlec B, Skrickova J, Merta Z, et al. The Incidence and Predictors of Thromboembolic Events in Patients with Lung Cancer. ScientificWorldJournal 2014;2014:125706.

4. Walker AJ, Baldwin DR, Card TR, et al. Risk of venous thromboembolism in people with lung cancer: a cohort study using linked UK healthcare data. Br J Cancer 2016;115:115-21.

5. Sørensen HT, Mellemkjaer L, Olsen JH, et al. Prognosis of cancers associated with venous thromboembolism. N Engl J Med 2000;343:1846-50.

6. Gussoni G, Frasson S, La Regina M, et al. Three-month mortality rate and clinical predictors in patients with venous thromboembolism and cancer. Findings from the RIETE registry. Thromb Res 2013;131:24-30.

7. Gary T, Belaj K, Steidl K, et al. Asymptomatic deep vein thrombosis and superficial vein thrombosis in ambulatory cancer patients: impact on short-term survival. Br J Cancer 2012;107:1244-8.

8. Chew HK, Wun T, Harvey DJ, et al. Incidence of venous thromboembolism and the impact on survival in breast cancer patients. J Clin Oncol 2007;25:70-6.

9. Key NS, Khorana AA, Kuderer NM, et al. Venous Thromboembolism Prophylaxis and Treatment in Patients With Cancer: ASCO Clinical Practice Guideline Update. J Clin Oncol 2020;38:496-520.

10. NCCN. NCCN Guidelines for Cancer-Associated Venous Thromboembolic Disease. 2020. Available online: https:// www.nccn.org/professionals/physician_gls/pdf/vte.pdf. Accessed 2020.8.12.

11. Spyropoulos AC, Eldredge JB, Anand LN, et al. External Validation of a Venous Thromboembolic Risk Score for Cancer Outpatients with Solid Tumors: The COMPASSCAT Venous Thromboembolism Risk Assessment Model. Oncologist 2020;25:e1083-90.

12. Mulder FI, Candeloro M, Kamphuisen PW, et al. The Khorana score for prediction of venous thromboembolism in cancer patients: a systematic review and meta-analysis. Haematologica 2019;104:1277-87.

13. Hachey KJ, Hewes PD, Porter LP, et al. Caprini venous thromboembolism risk assessment permits selection for postdischarge prophylactic anticoagulation in patients with resectable lung cancer. J Thorac Cardiovasc Surg 2016;151:37-44.e1.

14. Khorana AA, Soff GA, Kakkar AK, et al. Rivaroxaban for Thromboprophylaxis in High-Risk Ambulatory Patients with Cancer. N Engl J Med 2019;380:720-8.

15. Carrier M, Abou-Nassar K, Mallick R, et al. Apixaban to Prevent Venous Thromboembolism in Patients with Cancer. N Engl J Med 2019;380:711-9.

16. Agnelli G. Direct Oral Anticoagulants for Thromboprophylaxis in Ambulatory Patients with Cancer. N Engl J Med 2019;380:781-3.

17. Subramanian J, Govindan R. Molecular genetics of lung cancer in people who have never smoked. Lancet Oncol 2008;9:676-82.

18. Corrales-Rodriguez L, Soulières D, Weng X, et al. Mutations in NSCLC and their link with lung cancerassociated thrombosis: a case-control study. Thromb Res 2014;133:48-51.

19. Ng TL, Smith DE, Mushtaq R, et al. ROS1 Gene Rearrangements Are Associated With an Elevated Risk of Peridiagnosis Thromboembolic Events. J Thorac Oncol 2019;14:596-605.

20. Chiari R, Ricciuti B, Landi L, et al. ROS1-rearranged Non-small-cell Lung Cancer is Associated With a High Rate of Venous Thromboembolism: Analysis From a Phase II, Prospective, Multicenter, Two-arms Trial (METROS). Clin Lung Cancer 2020;21:15-20.

21. Dou F, Zhang Y, Yi J, et al. Association of ALK rearrangement and risk of venous thromboembolism in patients with non-small cell lung cancer: A prospective cohort study. Thromb Res 2020;186:36-41.

22. Al-Samkari H, Leiva O, Dagogo-Jack I, et al. Impact of ALK Rearrangement on Venous and Arterial Thrombotic Risk in NSCLC. J Thorac Oncol 2020;15:1497-506.

23. Dou F, Li H, Zhu M, et al. Association between oncogenic status and risk of venous thromboembolism in patients with non-small cell lung cancer. Respir Res 2018;19:88.

24. Verso M, Chiari R, Mosca S, et al. Incidence of Ct scandetected pulmonary embolism in patients with oncogeneaddicted, advanced lung adenocarcinoma. Thromb Res 2015;136:924-7.

25. Zer A, Moskovitz M, Hwang DM, et al. ALK-Rearranged Non-Small-Cell Lung Cancer Is Associated With a High Rate of Venous Thromboembolism. Clin Lung Cancer 2017;18:156-61.

26. Huang H, Korn JR, Mallick R, et al. Incidence of venous thromboembolism among chemotherapy-treated patients with lung cancer and its association with mortality: a retrospective database study. J Thromb Thrombolysis 2012;34:446-56.

27. Zugazagoitia J, Biosca M, Oliveira J, et al. Incidence, predictors and prognostic significance of thromboembolic disease in patients with advanced ALK-rearranged nonsmall cell lung cancer. Eur Respir J 2018;51:1702431. 
28. Alexander M, Pavlakis N, John T, et al. A multicenter study of thromboembolic events among patients diagnosed with ROS1-rearranged non-small cell lung cancer. Lung Cancer 2020;142:34-40.

29. Page MJ, McKenzie JE, Bossuyt PM, et al. Updating guidance for reporting systematic reviews: development of the PRISMA 2020 statement. J Clin Epidemiol 2021;134:103-12.

30. Smetana GW, Umscheid CA, Chang S, et al. Methods guide for authors of systematic reviews of medical tests: a collaboration between the Agency for Healthcare Research and Quality (AHRQ) and the Journal of General Internal Medicine. J Gen Intern Med 2012;27 Suppl 1:S1-3.

31. Jiang T, Su C, Li X, et al. EGFR TKIs plus WBRT Demonstrated No Survival Benefit Other Than That of TKIs Alone in Patients with NSCLC and EGFR Mutation and Brain Metastases. J Thorac Oncol 2016;11:1718-28.

32. Li X, Ren R, Ren S, et al. Peripheral blood for epidermal growth factor receptor mutation detection in non-small cell lung cancer patients. Transl Oncol 2014;7:341-8.

33. Wu C, Zhao C, Yang Y, et al. High Discrepancy of Driver Mutations in Patients with NSCLC and Synchronous Multiple Lung Ground-Glass Nodules. J Thorac Oncol 2015;10:778-83.

34. Alexander M, Solomon B, Burbury K. Thromboembolism in Anaplastic Lymphoma Kinase-Rearranged Non-Small Cell Lung Cancer. Clin Lung Cancer 2018;19:e71-2.

35. Azevedo S, Bei L, Cunha J, et al. Anaplastic Lymphoma Kinase Fusion Oncogene Positive Non-Small Cell Lung Cancer - The Experience of an Institution. J Thorac Oncol 2017;12:S1179.

36. Castellón Rubio VE, Luque Caro R, Gonzalez Flores $\mathrm{E}$, et al. Thromboembolic events (TE) in patients with metastatic non small cell lung cancer (NSCLC) EGFR/ ALK native treated with first-line platinum-based chemotherapy. Ann Oncol 2015;26:116.

37. Chen $M, X u$ Y, Zhao J, et al. Clinical Predictive Factors associated with First Line EGFR-TKI Efficacy in Advanced NSCLC Patients with EGFR Mutations. Zhongguo Fei Ai Za Zhi 2019;22:99-104.

38. Davidsson E, Murgia N, Ortiz-Villalón C, et al. Mutational status predicts the risk of thromboembolic events in lung adenocarcinoma. Multidiscip Respir Med 2017;12:16.

39. Gervaso L, Poudel SK, Roopkumar J, et al. Molecular Subtyping to Predict Risk of Venous Thromboembolism in Patients with Advanced Lung Adenocarcinoma: A Cohort Study. Blood 2019;134:3651.
40. Hiraide M, Shiga T, Minowa Y, et al. Identification of risk factors for venous thromboembolism and evaluation of Khorana venous thromboembolism risk assessment in Japanese lung cancer patients. J Cardiol 2020;75:110-4.

41. Itchins M, Alexander M, John T, et al. P2.01-50 Thromboembolism in ROS1 Rearranged Non-Small Cell Lung Cancer. J Thorac Oncol 2018;13:S684.

42. Yang S, Wang Q. ALK-Rearrangement May Promote VTE by Increasing the Expression of TF in Advanced Lung Adenocarcinoma. J Thorac Oncol 2019;14:S655.

43. Zer A, Joseph L, Battat E, et al. Venous thromboembolism (VTE) in patients with ALK-rearranged non-small cell lung cancer (ALK-LC): A population-based cohort. J Clin Oncol 2019;37:6608.

44. Khorana AA, Kuderer NM, Culakova E, et al. Development and validation of a predictive model for chemotherapy-associated thrombosis. Blood 2008;111:4902-7.

45. Howlett J, Benzenine E, Jonathan C, et al. Could venous thromboembolism and major bleeding be indicators of lung cancer mortality? A nationwide database study. BMC Cancer 2020;20:461.

46. Lyman GH, Culakova E, Poniewierski MS, et al. Morbidity, mortality and costs associated with venous thromboembolism in hospitalized patients with cancer. Thromb Res 2018;164:S112-8.

47. Key NS, Khorana AA, Mackman N, et al. Thrombosis in Cancer: Research Priorities Identified by a National Cancer Institute/National Heart, Lung, and Blood Institute Strategic Working Group. Cancer Res 2016;76:3671-5.

48. Rupa-Matysek J, Lembicz M, Rogowska EK, et al. Evaluation of risk factors and assessment models for predicting venous thromboembolism in lung cancer patients. Med Oncol 2018;35:63.

49. Ay C, Dunkler D, Marosi C, et al. Prediction of venous thromboembolism in cancer patients. Blood 2010;116:5377-82.

50. Lee A, Howell VM, Itchins M, et al. ROS1-Rearranged Non-Small-Cell Lung Cancer, Factor V Leiden, and Recurrent Venous Thromboses. Clin Lung Cancer 2018;19:457-9.

51. Gran OV, Smith EN, Brækkan SK, et al. Joint effects of cancer and variants in the factor 5 gene on the risk of venous thromboembolism. Haematologica 2016;101:1046-53.

52. Hoesel B, Mussbacher M, Dikorman B, et al. Androgen receptor dampens tissue factor expression via nuclear factor- $\mathrm{\kappa B}$ and early growth response protein 1. J Thromb Haemost 2018;16:749-58. 
53. Sen Y, Tang H, Wang Q. P1.01-017 ALK-Rearranged May Promote VTE by Increasing the Expression of TF in Advanced Lung Adenocarcinoma. J Thorac Oncol
2017;12:S1899.

(English Language Editors: D. Fitzgerald and J. Reynolds)

Cite this article as: Liu Y, Wang W, Wu F, Gao G, Xu J, Li X, Zhao C, Yang S, Mao S, Pan Y, Jia K, Shao C, Chen B, Ren S, Zhou C. High discrepancy in thrombotic events in non-small cell lung cancer patients with different genomic alterations. Transl Lung Cancer Res 2021;10(3):1512-1524. doi: 10.21037/ tlcr-20-1290 


\section{Supplementary}

Table S1 Quality evaluation of enrolled studies in meta-analysis.

\begin{tabular}{|c|c|c|c|c|c|c|c|c|c|c|c|c|}
\hline Reference & Year & (1) & (2) & (3) & (4) & (5) & (6) & (7) & (8) & (9) & (10) & (11) \\
\hline Hiraide et al & 2020 & 1 & 0 & 1 & 1 & 1 & 1 & 1 & 0 & 0 & 1 & 0 \\
\hline Dou et al & 2020 & 1 & 1 & 1 & 1 & 1 & 1 & 1 & 0 & 0 & 1 & 1 \\
\hline Chiari et al & 2020 & 1 & 1 & 0 & 1 & 1 & 1 & 0 & 0 & 0 & 1 & 0 \\
\hline Samkari et al & 2020 & 1 & 1 & 1 & 1 & 1 & 1 & 1 & 0 & 0 & 1 & 0 \\
\hline Alexander et al & 2020 & 1 & 1 & 1 & 1 & 1 & 1 & 0 & 0 & 0 & 1 & 0 \\
\hline $\mathrm{Ng}$ et al & 2019 & 1 & 1 & 1 & 1 & 1 & 1 & 1 & 0 & 0 & 1 & 0 \\
\hline Gervaso et al & 2019 & 1 & 1 & 1 & 1 & 1 & 0 & 0 & 0 & 0 & 1 & 0 \\
\hline Zer et al & 2019 & 1 & 1 & 1 & 1 & 1 & 1 & 0 & 0 & 0 & 1 & 0 \\
\hline Yang et al & 2019 & 1 & 1 & 1 & 1 & 1 & 0 & 0 & 0 & 0 & 1 & 0 \\
\hline Chen et al & 2019 & 1 & 1 & 1 & 1 & 1 & 1 & 1 & 0 & 0 & 1 & 0 \\
\hline Itchins et al & 2018 & 1 & 1 & 1 & 1 & 1 & 0 & 0 & 0 & 0 & 1 & 0 \\
\hline Zugazagoitia et al & 2018 & 1 & 1 & 1 & 1 & 1 & 1 & 1 & 0 & 0 & 1 & 0 \\
\hline Dou et al & 2018 & 1 & 1 & 1 & 1 & 1 & 1 & 1 & 0 & 0 & 1 & 0 \\
\hline Alexander et al & 2018 & 1 & 1 & 1 & 1 & 1 & 0 & 0 & 0 & 0 & 1 & 0 \\
\hline Davidsson et al & 2017 & 1 & 1 & 1 & 1 & 1 & 1 & 0 & 0 & 0 & 1 & 0 \\
\hline Azevedo et al & 2017 & 1 & 1 & 1 & 1 & 1 & 1 & 0 & 0 & 0 & 1 & 0 \\
\hline Zer et al & 2016 & 1 & 0 & 1 & 1 & 1 & 1 & 1 & 0 & 0 & 1 & 0 \\
\hline Castellón et al & 2015 & 1 & 1 & 1 & 1 & 1 & 0 & 0 & 0 & 0 & 1 & 0 \\
\hline Corrales-Rodriguez et al & 2014 & 1 & 1 & 1 & 1 & 1 & 1 & 1 & 0 & 0 & 1 & 0 \\
\hline Verso et al. & 2014 & 1 & 1 & 1 & 1 & 1 & 0 & 0 & 0 & 0 & 1 & 0 \\
\hline
\end{tabular}

1) Defines the source of information (e.g., survey, record review).

2) Lists inclusion and exclusion criteria for exposed and unexposed subjects (cases and controls) or refer to previous publications.

3) Indicates time period used for identifying patients.

4) Indicates whether or not subjects were consecutive if not population-based.

5) Indicates if evaluators of subjective components of study were masked to other aspects of the status of the participants.

6) Describes any assessments undertaken for quality assurance purposes (e.g., test/retest of primary outcome measurements).

7) Explains any patient exclusions from analysis.

8) Describes how confounding was assessed and/or controlled.

9) If applicable, explains how missing data were handled in the analysis.

10) Summarizes patient response rates and completeness of data collection.

11) Clarifies what follow-up, if any, was expected and the percentage of patients with incomplete data or follow-up. 
A study

Omitting Hiraide et al Omitting Dou et al Omitting Chiari et al
Omitting Samkari et Omitting Alexander et al Omitting Ng et al Omitting Gervaso et Omitting Zer et al Omitting Yang et Omitting Chen et al Omitting Itchins et al Omitting Zugazagoitia et Omitting Dou et al Omitting Alexander et al
Omitting Davidsson et a Omitting Azevedo et at Omitting Zer et al

Omitting Castellon et al Omitting Corrales-Rodriguez et Omitting Verso et al

Random effects mode

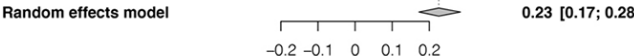

C

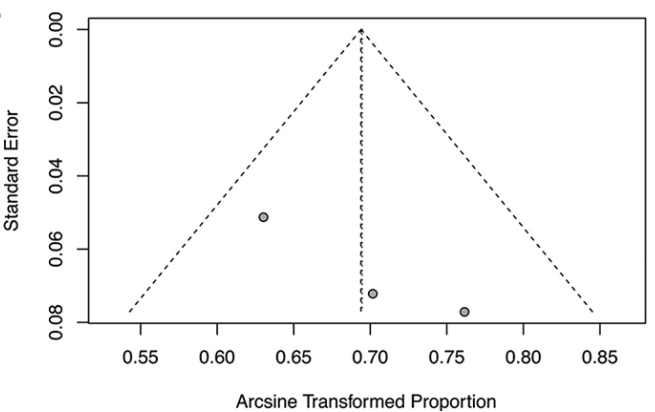

B

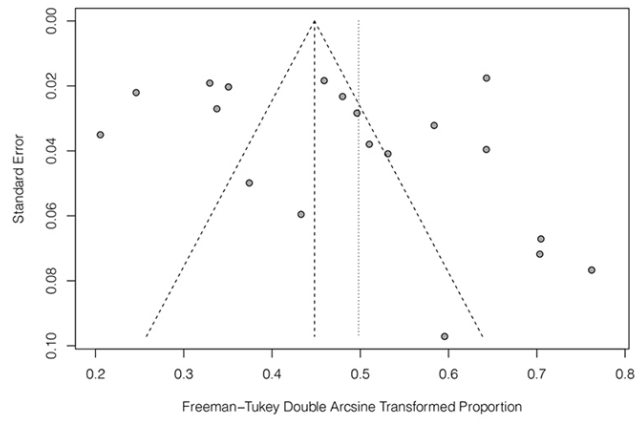

Figure S1 Sensitivity and publication bias of enrolled studies. (A) Sensitivity analysis of all the studies enrolled. (B) Funnel plot of publication bias of total studies. (C) Funnel plot of publication bias of total studies in the subgroup meta-analysis of ROS1-fusion non-small cell lung cancer.
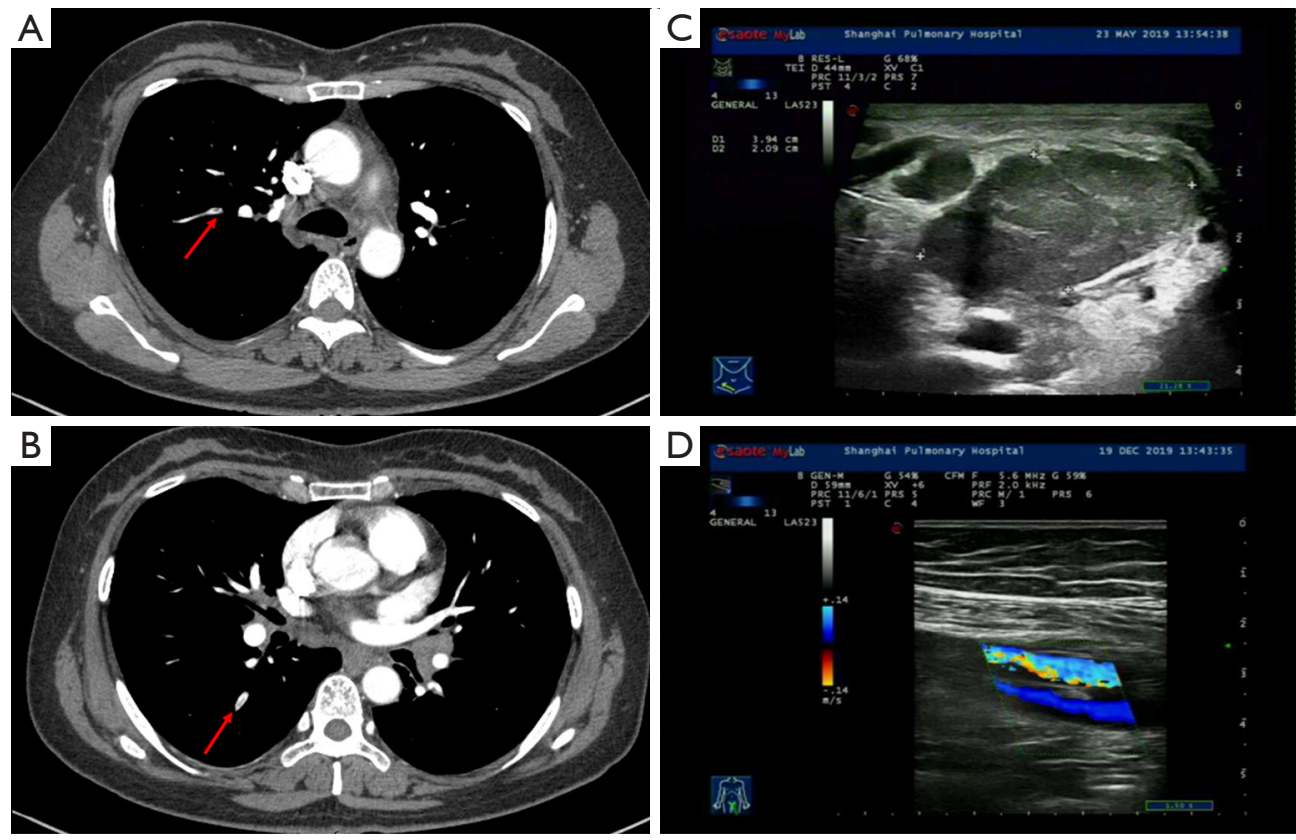

Figure S2 Representative images of ultrasound and computed tomography for the diagnosis of pulmonary embolism or deep vein thrombosis. (A) pulmonary embolism; (B) pulmonary embolism; (C) Jugular vein thrombosis; (D) Femoral vein thrombosis. 
A

Crizotinib

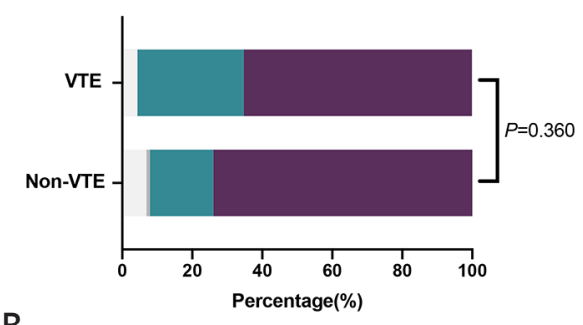

B

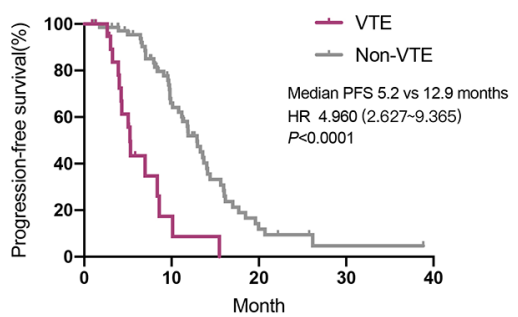

C

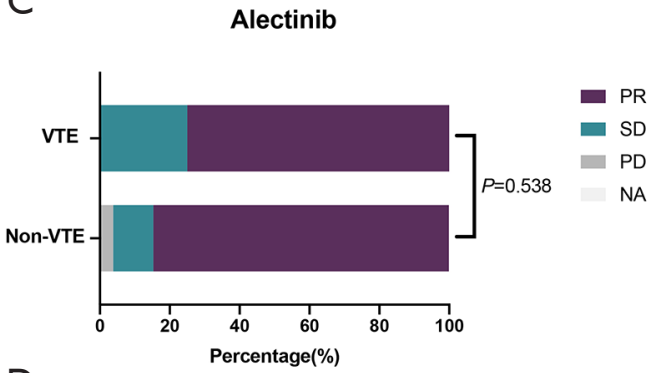

D

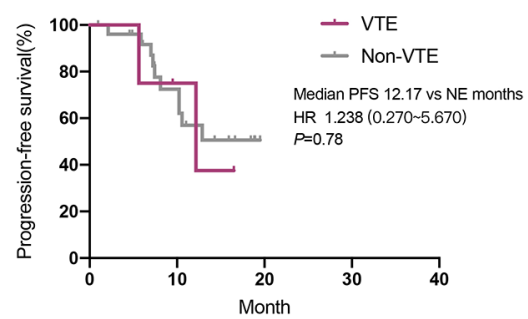

Figure S3 Subgroup analyses of PFS in ALK-fusion patients treated with crizotinib or alectinib. (A) Response to crizotinib; (B) PFS of patients treated with crizotinib; (C) response to alectinib; (D) PFS of patients treated with alectinib. ALK, anaplastic lymphoma kinase; PFS, progression-free survival. 